\title{
Dealing with imprecise information in group multicriteria decisions: a methodology and a GDSS architecture
}

\author{
Luis C. Dias *, João N. Clímaco \\ INESC Coimbra and Faculty of Economics, University of Coimbra, Av. Dias da Silva 165, 3004-512 Coimbra, Portugal
}

Received 27 September 2002; accepted 24 July 2003

Available online 6 December 2003

\begin{abstract}
This paper outlines a distributed GDSS suitable to be used over the Internet, based on the VIP Analysis methodology and software. VIP Analysis incorporates complementary approaches to deal with the aggregation of multicriteria performances by means of an additive value function under imprecise information. This proposed GDSS intends to support a decision panel forming a democratic decision unit, whose members wish to reach a final decision in a choice problem, based on consensus or on some majority rule. Its purpose is not to impose an aggregated model from the individual ones. Rather, the GDSS is designed to reflect to each member the consequences of his/her inputs, confronting them with analogous reflections of the group members' inputs. We propose aggregation procedures to provide a reflection of the group's inputs to each of its members, and an architecture for a GDSS implementing these procedures.
\end{abstract}

(C) 2003 Elsevier B.V. All rights reserved.

Keywords: Decision support systems; Group decisions and negotiation; Multicriteria analysis; Imprecise information

\section{Introduction}

Group decision processes and negotiation processes constitute very complex human activities. Improving their understanding justifies multidisciplinary studies, based on cognitive psychology, organizational science, sociology, political science, etc. Furthermore, the development of computer-based support tools for these activities is rooted not only in communication/information technologies and OR/management science models, but also in human and organizational behavior studies.

This paper discusses how an existing standalone decision support tool - VIP Analysis (Dias and Clímaco, 2000a) - may evolve to become a distributed group decision support system (GDSS) suitable to support decision making via the Internet. We address the extension to a group decision-making setting of the VIP Analysis methodology, and propose a GDSS architecture for the extension of the VIP Analysis software.

\footnotetext{
${ }^{*}$ Corresponding author. Tel.: +351-39-32689; fax: +351-39-24692.

E-mail addresses: 1dias@inescc.pt (L.C. Dias), jclimaco@inescc.pt (J.N. Clímaco).
} 
VIP Analysis deals with the aggregation of multicriteria performances by means of an additive value function (e.g. see Keeney and Raiffa, 1976), in the context of choosing the best alternative in a set. It is designed to accept imprecise information (in the sense of Athanassopoulos and Podinovski, 1997; Weber, 1987) on the model's scaling constants when the Decision Makers (DMs) are not able to or do not wish to provide precise values (for a general classification of uncertainty sources and approaches see Zimmermann, 2000). To deal with such cases, VIP Analysis considers the model's scaling constants (which reflect the importance of the criteria) as interdependent variables that may take several values subject to constraints. The DMs, or the analyst (when exists), will find several complementary approaches in a single package, rather than having to use different programs for different approaches.

Group decision making may benefit from working with imprecise information for many reasons. First, it is easier for each DM, individually, to provide constraints or bounds on the variables than to find the "most correct" value for them; from another perspective, it is easier for an analyst to elicit constraints than precise values. Second, exploring the results that are known to hold despite the imprecision (the "robust" conclusions) provides hints to drive the group's discussion. Third, DMs avoid committing too early to precise values, which might cause premature (and sometimes unnecessary) conflict. Rather, they may start by agreeing on loose constraints and try to maintain that consensus atmosphere as they converge to more precise results. Often, different input values lead to the same conclusions. The purpose of VIP Analysis is hence to allow the DMs to gain insight on the situation and to let them progress in the decision process without demanding from them precise values for the parameters, progressively reducing the number of alternatives.

Although standalone VIP Analysis may be a useful tool in some group decision contexts, namely in faceto-face full cooperative settings, we are aware of its many limitations. An important limitation is that it lacks the capabilities present in most GDSS's, which allow DMs to perform private analyses and to communicate electronically. Among other implications, this excludes its use when the DMs are spatially distributed. A second important limitation is that VIP Analysis assumes that a model is given, which implicitly aggregates the different models that the DMs may have (here, a model corresponds to a set of acceptable combinations of parameter values).

The proposed extension of the VIP Analysis software to a multiperson use (VIP-G, for short) combines computer-mediated communication with an adaptation (to a collaborative group environment) of the VIP Analysis multiattribute evaluation tools, regarding the reconciliation of conflicts via a certain kind of cognitive feedback (Bose and Paradice, 1999).

As stated by Davey and Olson (1998) "Decision making groups can range from cooperative, with very similar goals and outlooks, to antagonistic, with diametrically opposed objectives. Even in cooperative groups, conflict can arise during the decision process". Roughly speaking, in this paper we are concerned with group decision, rather than with negotiation. However, the frontier between them is fuzzy.

Conflict settings where the parties involved have fundamentally opposed objectives (e.g., buyer/seller) are excluded from the scope of VIP-G, but settings where the priorities of the group's members may diverge, such as in resource allocation problems, are not excluded. VIP-G is designed to support a decision panel (e.g., a board of directors, a quality circle, the jury of a competition. ..) forming a democratic decision unit whose members wish to reach a final decision, in a choice problem, based on consensus or on some majority rule. The reconciliation of the different points of view of the group members is essentially supported by a multiattribute additive model adequate to deal with imprecise information on some of the parameters, together with appropriate tools helping to evaluate and/or guide the individual/group behavior, and with adequate human/computer interface devices. The architecture and the aggregation tools of this distributed GDSS are designed to contribute to high levels of cohesiveness and performance, as well as equality of participation opportunities among a disperse group of actors, communicating via Internet in synchronous or asynchronous settings, depending on the circumstances. 
We will now typify what we mean by group decision in opposition to negotiation processes, discussing some major contrasting characteristics. First, the fundamental objectives are common in group decisions, while in negotiation they are opposed. Secondly, the set of alternatives is enumerated in group decisions (although it can evolve during the communication/decision process), while in negotiation alternatives (proposals) are sequentially presented by the parties and are to a great extent interactively generated, making concessions and/or accommodating some needs of the other parties. This peculiar interdependence among actors "rather than conflict, distinguishes negotiation from other forms of decision making" (Kersten and Cray, 1996). Thirdly, in group decisions constraints are discussed, sharing information in order to foster interactive learning among the actors, profiting of their own experiences and of their diversified cultural roots, and contributing to reduce uncertainty and ambiguity, while in negotiation constraints are hidden (e.g., a sales target, or a purchase budget). Furthermore, in negotiation the values and beliefs of the parties are also hidden, being frequently unconsciously adopted. Fourthly, voting is a possibility of setting divergence in group decisions, but not in negotiations, where mediation or arbitration may be needed to approximate the parties. Negotiation involves competition while group decisions are mostly based in deliberative processes. Finally, leaving the group is not usually an option in group decisions, while abandoning the negotiation ("walking away") is often an option. In fact, the quality of the decisions of a group strongly depends on the cohesion of the group.

The remainder of this paper is structured as follows. The next section presents the methodology of VIP Analysis and its extension, and discusses how it may be used in group decision settings. Section 3 introduces an illustrative example. In Section 4 we propose an architecture for a GDSS intended to support distributed decision making, based on the methodology presented before. Finally, we offer some conclusions and indicate some future research paths.

\section{Methodology}

\subsection{Basic VIP analysis concepts}

We will first overview the current VIP Analysis methodology and software (Dias and Clímaco, 2000a, describe it in more detail). The purpose of VIP Analysis is to support the evaluation of a discrete set of alternatives, in order to choose the most preferred one, according to a multiattribute additive value function (Keeney and Raiffa, 1976). The global value of an alternative $a_{i}$ is a sum of its values at the $n$ criteria $\left(v_{1}\left(a_{i}\right), \ldots, v_{n}\left(a_{i}\right)\right)$, weighted by $n$ scaling constants $k=\left(k_{1}, \ldots, k_{n}\right)$ that indirectly reflect the importance of the criteria:

$$
V\left(a_{j}, k\right)=\sum_{j=1}^{n} k_{j} v_{j}\left(a_{j}\right), \quad \text { with } \sum_{j=1}^{n} k_{j}=1 \text { and } k_{j} \geqslant 0 .
$$

One of the most difficult parts of the decision aid process is setting the values of the scaling constants, since these parameters will reflect the DMs' values and trade-offs (e.g., how much would you be willing to lose in attribute "cost" to gain one unit in attribute "risk of accident"?). Indeed, not only DMs may find it hard to provide precise figures about their preferences, but also these preferences may change as the decision aid process evolves. Moreover, the procedures used to elicit the values of the scaling constants (e.g., see Von Winterfeldt and Edwards, 1986) may require more time and patience than some DMs can spare. Finally, in group decision situations the opinions and preferences of the DMs diverge frequently.

To overcome these difficulties, VIP Analysis proposes to advance in the decision process with variable interdependent parameters. This means that instead of requiring precise values for the scaling constants, it asks for intervals and linear constraints on these values. For instance, a DM (or a group of DMs) may be 
doubtful about setting $k_{1}=0.2$ and $k_{2}=0.1$ (these precise values) but may find it easy to agree that $k_{1}>k_{2}$. This kind of information is often designated as poor, imprecise, incomplete, or partial information (e.g., see Weber, 1987; Dias and Clímaco, 2000a). The constraints usually stem from imprecise answers from the DM (e.g. providing an interval for the trade-off rate between two criteria) or from holistic judgments about alternatives that the DM is able to compare (e.g. $a_{1}$ is preferred to $a_{2}$ ). When VIP Analysis is used to support a fully cooperative group of DMs meeting face to face, reaching a consensus on the constraints to insert in the model can be difficult. However, we argue that reaching a consensus on precise values is much more difficult.

Let $T$ denote the set of all combinations of parameter values that satisfy all the constraints inserted. We assume that the system of constraints would be consistent; otherwise, $T$ would be an empty set. Once $T$ is defined, VIP Analysis may be used to discover robust conclusions (those that hold for every combination in $T$ ) and to identify which results are more affected by the imprecision in the parameter values (the results that vary more). The results produced by VIP Analysis from a set $T$ of acceptable combinations of values for the importance parameters and a set $A=\left\{a_{1}, \ldots, a_{m}\right\}$ of alternatives include the following:

- A range of values for each alternative $a_{i} \in A$, from $\min \left\{V\left(a_{i}, k\right): k \in T\right\}$ to $\max \left\{V\left(a_{i}, k\right): k \in T\right\}$. If the highest possible value for an alternative $a_{x}$ is always less than the lowest possible value for an alternative $a_{y}$, then the first alternative could be discarded.

- The highest difference of value for each ordered pair of alternatives $\left(a_{i}, a_{j}\right) \in A^{2}$, i.e., $\max \left\{V\left(a_{i}, k\right)-V\left(a_{j}, k\right): k \in T\right\}$. If the maximum difference is negative or null then $V\left(a_{j}, k\right) \geqslant V\left(a_{i}, k\right)$ $\forall k \in T$, which we denote as $a_{j} \Delta a_{i}\left(a_{j}\right.$ "dominates" $\left.a_{i}\right)$. If the maximum difference does not exceed a tolerance parameter $\varepsilon$, then $V\left(a_{j}, k\right) \geqslant V\left(a_{i}, k\right)-\varepsilon \forall k \in T$, which we denote as $a_{j} \Delta_{\varepsilon} a_{i}\left(a_{j}\right.$ "quasi-dominates" $a_{i}$ with tolerance $\varepsilon$ ).

- For each alternative $a_{i} \in A$, the "maximum regret" associated with choosing it, i.e. the maximum difference of value considering the alternatives that may have a higher value than $a_{i}$ given $T$ :

$$
\begin{aligned}
\operatorname{Regret}_{\max }\left(a_{i}\right) & =\max \left\{\operatorname{Regret}\left(a_{i}, k\right): k \in T\right\} \quad(i=1, \ldots, m) . \\
& =\max \left\{\max \left\{V\left(a_{j}, k\right): j=1, \ldots, m\right\}-V\left(a_{i}, k\right): k \in T\right\} \quad(i=1, \ldots, m) .
\end{aligned}
$$

If Regret $\max _{\max }\left(a_{i}\right) \leqslant 0$ then we can say that $a_{i}$ is "optimal"; if $\operatorname{Regret}_{\max }\left(a_{i}\right) \leqslant \varepsilon$ we can say that $a_{i}$ is "quasioptimal" with tolerance $\varepsilon$.

\subsection{Extension of the methodology for explicit aggregation of individual views}

VIP Analysis, as presented in the previous section, can be a useful tool to support a group of DMs meeting face-to-face around a computer, sharing an imprecisely defined model, therefore agreeing on the robust conclusions. The outputs of VIP Analysis may allow the decision process to progress, as the DMs learn about the model and the problem, postponing the elicitation questions they find difficult to answer, or prone to generate conflict. For instance, they may not agree on precise values for the scaling constants, but may agree on a ranking of those constants (e.g. $k_{1} \geqslant k_{2} \geqslant k_{3} \geqslant k_{4}$ ). Or they may merely agree on a partial ranking (e.g. $k_{1} \geqslant k_{2} ; k_{1} \geqslant k_{3}$ and $k_{3} \geqslant k_{4}$ ). In any case, VIP Analysis provides results that may allow to eliminate some alternatives or to highlight the most promising ones. Furthermore, the results can be used to direct the elicitation of further information with the purpose of progressively reducing the imprecision (e.g. "can alternative $a_{x}$ really attain such a high value?" "can all DMs agree that $a_{x}$ is worse than $a_{y}$ ?").

Nevertheless, we are aware of the limitations of VIP Analysis as a standalone tool. Namely, it excludes settings where the DMs are spatially distributed, it does not allow DMs to analyze the problem individually, and it implicitly requires an a priori aggregation of the different models that the DMs may have. We will now address methodological extensions concerning aggregation support. 
VIP Analysis may be seen as a tool that analyses the consequences in terms of output of the imprecise information taken as input. In particular, it is not normative to a point where it recommends which alternative is the best one given the inputs. In a group decision context, we intend to keep this attitude, meaning that a GDSS based on VIP Analysis should neither propose (upon aggregating) a final ranking of the alternatives, nor impose an aggregated model from the individual ones. Rather, the GDSS will be designed to reflect (like a mirror) to each member the consequences of his/her inputs, confronting them with analogous images of the group members' inputs. The aggregation procedures will hence be used only to provide a reflection of the group's inputs to each of its members, showing all the results that are compatible with the inputs provided, but without pointing to a particular result as being the group's choice.

Let us consider a group of $K \mathrm{DMs}$ who are collaborating to recommend a solution to a decision problem, each one having a set $T_{k}$ of acceptable values for the parameters $(k=1, \ldots, K)$. Individual perspectives may be aggregated either at the input level or at the output level. When aggregation occurs at the input level (Fig. 1), an operator $f(\cdot)$ aggregates the individual $T_{k}(k=1, \ldots, K)$ into a set $T$ of values accepted by the group, whereas an operator $e(\cdot)$ yields all the results of the method $R$ compatible with $T$. When aggregation occurs at the output level (Fig. 2), the operator $e(\cdot)$ yields the set of results of the method compatible with each DM's $T_{k}$, whereas an operator $h(\cdot)$ aggregates the individual sets of results $R_{k}$ into a set of results $R$. The aggregation performed by $f(\cdot)$ and $h(\cdot)$ does usually consist in averaging, minimizing a distance measure, voting, etc.

\subsection{A review of previous research in a similar setting}

Before trying to explore the consequences of imprecision in the framework of the aggregation support for group settings using VIP Analysis, we will now outline some previously proposed approaches. Taking into account the context of VIP analysis, this review is limited to approaches based on additive value functions and imprecise information for group decision making.

Kim and Ahn (1997) propose a two steps approach. The first step is based on the imprecise (or incomplete) information provided by the decision makers and consists in the articulation of their preferences leading to individual results. This is based on the calculation of maximum/minimum expected values, strict/weak dominance values, or ordinal ranking. In a second step a procedure to aggregate the preferences of all group members is proposed, where the aggregation function is a difference of weighted sums of each pair of actions, i.e., the group consensus is pursued from the analysis of pairwise dominance relations between pairs of alternatives.

Kim and Ahn (1999) propose another approach starting again by the calculation of individual results, i.e. strict or weak dominance relations are calculated by solving a series of LP problems. Here the utility

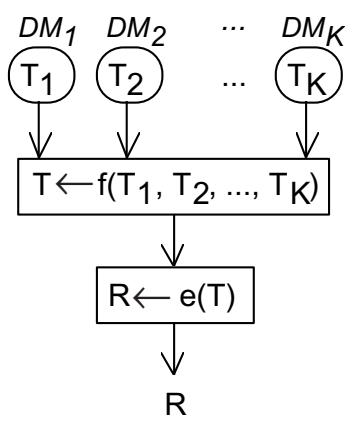

Fig. 1. Individual perspectives aggregated at the method's input level. 


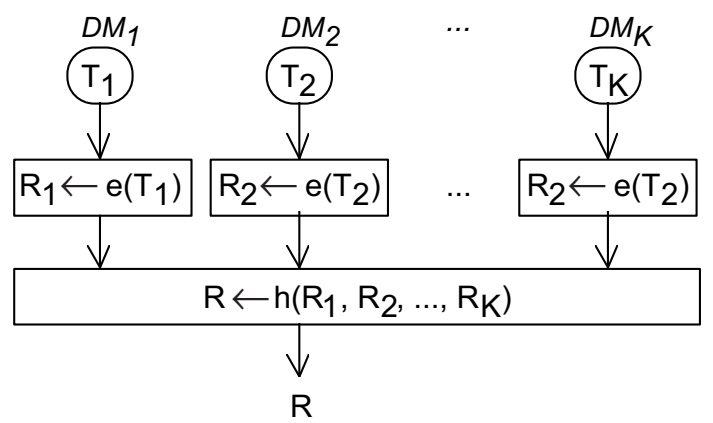

Fig. 2. Individual perspectives aggregated at the method's output level.

function which aggregates the individual results is approached using as inputs the individual weak dominance relations and it is built taking explicitly into account the individual preference strength. In fact, it is based on the net preference intensity of each alternative defined as the difference between the degree a alternative is preferred to all the others and that of the others being preferred to it.

Kim and Han (1999) propose a different approach. They assume imprecise information in the individual utilities and in the weights associated to each additive value function as well as in the weights related to a group utility function. Also here in a first step preferences are aggregated through weak/strict dominance relations based on the calculation of $\mathrm{max} / \mathrm{min}$ expected values. It is suggested the use of a probabilistic measure, which is an indicator for the preference strength between alternatives. A second step, dedicated to the enrichment of the group consensus, is also based on dominance relations. The elicitation of complementary information from the decision makers required by the second step is made interactively and facilitated by the probabilistic measure above referred to. A mathematical programming model is proposed to obtain the dominance relations.

Kim et al. (1999) propose still another approach to situations where the available information is imprecise. Here, a range-type representation for utilities is used. In a first step, the utility range for each member of the group is obtained by maximizing and minimizing, subject to constraints, the individual utility functions. In a second step, the aggregation of the individual ranges yields the so-called total range and agreed range of the group's preferences. The first corresponds to the intersection and the second to the union of the group members' ranges. Then, the decision makers try interactively to reduce the gap between total range and agreed range. Finally, a weak domination relation based on pairwise comparison is built. If any of the decision makers is not yet satisfied, the process is repeated taking into account the information elicited previously.

Bana e Costa (1986) considers, in the framework of additive value functions, the actors express different, and usually conflicting, levels of acceptance for the weights and proposes a methodology to enable the decision maker to take into account these differences in the evaluation of the relative importance of the criteria. Quoting the author: "The methodology proposes an Overall Compromise Criterion which "corrects" the average sum aggregation of the performances by an Acceptability Index combining all actors individual preferences". The group Acceptability Index is calculated using a joint density function. The process enables to identify the least conflicting alternative.

Bana e Costa (1990) considers imprecise information regarding the different actors criterion preference information. He proposes a two steps approach. First, the preferences representation is based on the definition of outranking relations. Secondly, the incomparability between pairs of alternatives is progressively reduced, in a compensatory framework. Thus, the use of the concept of Acceptability Index (Bana e Costa, 1986), is combined with a new outranking approach designated by the author as Outweight Analysis. 
Lahdelma et al. (1998) developed SMAA - stochastic multiobjective acceptability analysis. This approach is adequate to group decision settings, and considers that inaccurate or uncertain inputs can be represented as probability distributions. Key issues of this method are: exploring the weight space, an extension of the concept of acceptability index for each alternative (Bana e Costa, 1986), measuring the variety of the valuations supporting it, together with the definition of a central weight vector for each alternative, as the centre of gravity of weight space sub-region associated to it. The idea is to identify good compromise alternatives in the framework of jointly accepted (by the DMs) additive utility functions shape. Furthermore, a Confidence Factor is used in order to conclude about the level of accuracy of the input data for supporting a decision. In Hokkanen et al. (1998) and in Lahdelma and Salminen (2001) extensions of the SMAA are proposed.

In most of the approaches presented here, the explicit calculation of the individual results precedes the search of group aggregated results (and this can be obtained by the application of an operator, previously designated by $h(\cdot)$ ). Some of these approaches result in a ranking of the alternatives, whereas others are not so normative.

\subsection{Methodology behind VIP-G}

Keeping VIP Analysis' attitude of exploring the consequences of imprecision, it seems appropriate to consider $f(\cdot)$ or $h(\cdot)$ (Figs. 1 and 2 ) as the set operations $\cap$ (intersection) or $\cup$ (union), as proposed by Dias and Clímaco (2000b) for the context of Electre TRI. These are adequate operators as they do not perform any kind of voting calculus: They just state what everybody agrees to include and what everybody agrees to exclude, i.e., what inputs (or outputs) generate consensus. Other operators involve some kind of voting using cardinal or ordinal preference information.

When using intersections in $f(\cdot)(h(\cdot))$, set $T$ (set $R$, respectively) will gather the input values (results, respectively) that are acceptable by all the DMs, whereas if the union operation is used in $f(\cdot)(h(\cdot))$, the DMs will know the input values (results) that are not considered by any of them. In the first case, there is consensus on what to include; in the second case, there is consensus on what to exclude.

Extending these possibilities, we also consider an " $\alpha$-majority" aggregation operator, where a combination of parameter values or a result are considered acceptable if accepted by at least $\alpha K$ DMs (formally, this can be seen as a continuous-space extension of Aizerman and Aleskerov's (1983) "k-majority" operators for social choice functions). This is equivalent to voting, for every conceivable input (or result) if the group accepts it or not. The parameter $\alpha$ may vary between $1 / K$ (corresponding to the operator $\cup$ ) and 1 (corresponding to the operator $\cap$ ), possibly in increments of $1 / K$. Indeed, if there are four DMs, the required "majorities" can be $1 / 4,2 / 4,3 / 4$ and $4 / 4$ : Any value in the interval $[1 / 4,2 / 4[$ yields the same as a value of $1 / 4$, etc.

If the aggregation is performed at the input level (Fig. 1), a possible " $\alpha$-majority" aggregation operator could be

$$
T_{(\alpha)}=f_{\alpha}\left(T_{1}, \ldots, T_{K}\right)=\left\{t \in \bigcup_{k=1}^{K} T_{k}: \frac{\#\left\{k \in\{1, \ldots, K\}: t \in T_{k}\right\}}{K} \geqslant \alpha\right\} \quad(\alpha \in[1 / K, 1]) .
$$

This means that a combination $t$ (a vector) of parameter values is considered acceptable (i.e. as belonging to $\left.T_{(\alpha)}\right)$ if at least $\alpha \cdot K$ DMs include it in their acceptable sets. As a particular case, when $\alpha=1$, then $t \in T_{\alpha}$ if all DMs include $t$ in their sets. When $\alpha=1 / K$, then for $t \in T_{(\alpha)}$ to hold it is enough that one DM includes $t$ in his/her set. Hence

$$
f_{1}\left(T_{1}, \ldots, T_{K}\right)=\bigcap_{k=1}^{K} T_{k} \quad \text { and } \quad f_{1 / K}\left(T_{1}, \ldots, T_{K}\right)=\bigcup_{k=1}^{K} T_{k} .
$$


The purpose of computing $T_{(\alpha)}$ is not to impose a "consensus" model to be used thereafter, but to provide some feedback to the individual DMs, who may confront their private model with models acceptable by the group (by all members if $\alpha=1$, by at least one member if $\alpha=1 / K$ ). However, since the union of polyhedral sets is not usually a polyhedron, $T_{(\alpha)}(\alpha>1 / K)$ would not be a valid input for VIP Analysis. Hence, a more appropriate choice would be to take the convex hull of $T_{(\alpha)}$ instead. Unfortunately, the computations to compute the convex hull may be complex and time consuming (Avis et al., 1997). It may happen that $T_{(\alpha)}$ becomes void, particularly as $\alpha$ approaches 1 , although this should not happen frequently if there is no strong conflict among the DMs. In such cases, the individual polytopes might be changed either by discussing the constraints or by automatically dropping or loosening constraints in a fair manner.

Performing the aggregation at the output level (Fig. 2) is simpler and, since there is a many-to-one correspondence between parameter values and results (e.g., there are many combinations of parameter values yielding the same alternative as the best one), it is more likely to facilitate consensus. A possible " $\alpha$ majority" aggregation operator could be

$$
R_{(\alpha)}=h_{\alpha}\left(R_{1}, \ldots, R_{K}\right)=\left\{r \in \bigcup_{k=1}^{K} R_{k}: \frac{\#\left\{k \in\{1, \ldots, K\}: r \in R_{k}\right\}}{K} \geqslant \alpha\right\} \quad(\alpha \in[1 / K, 1]) .
$$

This means that a result $r$ (a number) is considered acceptable (i.e. as belonging to $R_{(\alpha)}$ ) if at least $\alpha \cdot K$ DMs include it in their result sets. As particular cases, we have $\alpha=1$ (all DMs include it) and $\alpha=1 / K$ (at least one DM includes it):

$$
h_{1}\left(R_{1}, \ldots, R_{K}\right)=\bigcap_{k=1}^{K} R_{k} \text { and } h_{1 / K}\left(R_{1}, \ldots, R_{K}\right)=\bigcup_{k=1}^{K} R_{k} .
$$

It may happen that $R_{(\alpha)}$ becomes void, which implies that $T_{(\alpha)}$ is also void. However, there may exist situations where $T_{(\alpha)}$ is void but not $R_{(\alpha)}$. The purpose of computing the set $R_{(\alpha)}$ is not to impose a "consensus" set of results, but to provide some feedback to the individual DMs, who may confront their private results with the ones accepted by the group (all the members if $\alpha=1$, at least one member if $\alpha=1 / K$, or some required support in between this extremes).

The conclusions that are drawn from $R_{(\alpha)}$ need some careful interpretation. For instance, consider a group composed of three DMs evaluating an alternative $a_{i}$. The first DM accepts a convex set of possible input values $\left(T_{1}\right)$, which (let us suppose) yield $\min \left\{V\left(a_{i}, k\right): k \in T\right\}=0.4$ and $\max \left\{V\left(a_{i}, k\right)\right.$ : $k \in T\}=0.65$. From the convexity of $T_{1}$ and the linearity of $V(\cdot)$, the interval of possible results corresponding to $T_{1}$ is $V\left(a_{i}\right) \in[0.4,0.65]$. Let us further suppose that the set $T_{2}$ of possible input values accepted by the second DM yielded the interval $V\left(a_{i}\right) \in[0.5,0.7]$, whereas the third DM reached the interval $V\left(a_{i}\right) \in[0.6,0.75]$ (see Fig. 3). In this case, $R_{(1)}$ would yield that $V\left(a_{i}\right) \in[0.6,0.65]$ (i.e. values acceptable by all DMs), $R_{(2 / 3)}$ would yield that $V\left(a_{i}\right) \in[0.5,0.7]$ (i.e. values acceptable by at least 2 out of $3 \mathrm{DMs}$ ), and $R_{(1 / 3)}$ would yield that $V\left(a_{i}\right) \in[0.4,0.75]$ (i.e. values acceptable by at least one DM). Now, it is not reasonable to say that the group unanimously considers that $V\left(a_{i}\right)$ is not less than 0.6 , just because the intersection $R_{(1)}$ yields that $V\left(a_{i}\right) \in[0.6,0.65]$. Indeed, only the third DM supports that conclusion. What we could say (from $\left.R_{(1 / 3)}\right)$ is that the group unanimously agrees that $V\left(a_{i}\right)$ cannot be less than 0.4 .

This approach to aggregating the outputs may hence be seen from another perspective while keeping the same idea. Let us consider $C_{k}(k=1, \ldots, K)$ as sets of individual conclusions (propositions) of the following types: $V\left(a_{i}\right) \geqslant x ; V\left(a_{i}\right) \leqslant x$; Regret $\left(a_{i}\right) \leqslant x$; and $V\left(a_{i}\right)-V\left(a_{j}\right) \leqslant x$.

The " $\alpha$-majority" aggregation operator $(\alpha \in[1 / K, 1])$ yields a set of conclusions acceptable by the group as follows: 


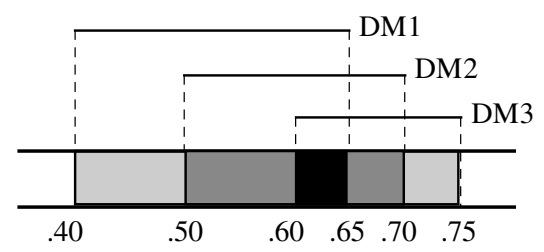

Fig. 3. Range of value for $a_{i}$ according to three members of a group.

$$
C_{(\alpha)}=q_{\alpha}\left(C_{1}, \ldots, C_{K}\right)=\left\{c \in \bigcup_{k=1}^{K} C_{k}: \frac{\#\left\{k \in\{1, \ldots, K\}: c \in C_{k} \vee\left(c^{\prime} \Rightarrow c \wedge c^{\prime} \in C_{k}\right)\right\}}{K} \geqslant \alpha\right\} .
$$

This means that a conclusion $c$ (a proposition) is considered acceptable (i.e. as belonging to $C_{(\alpha)}$ ) if at least $\alpha \cdot K \mathrm{DMs}$ reach the same conclusion (or a conclusion that implies it; e.g., the conclusion $V\left(a_{i}\right) \geqslant 0.8$ implies the conclusion $V\left(a_{i}\right) \geqslant 0.7$ ). As particular cases, we have $\alpha=1$ (all DMs reach a conclusion) and $\alpha=1 / K$ (at least one DM reaches it).

There is a direct correspondence (a sort of duality) between sets $C_{(\alpha)}$ and $R_{(1-\alpha+1 / K)}$ : the consequences in the first are drawn from the results in the latter, and vice versa. In particular, if we know from $R_{(1 / K)}$ that for a given variable $v(\cdot)$ all values in an interval $[x, y]$ are acceptable by at least one DM, then we will know that the conclusions " $v(\cdot)$ cannot be lower than $x$ " and " $v(\cdot)$ cannot be higher than $y$ " are robust conclusions accepted by all the group (i.e. belong to $C_{(1)}$ ).

It may also be of interest for a member to determine the aggregated results of a sub-group $G$. Namely, $G$ may correspond to the group formed by all members except him/her, or to a group of members that he/she perceives as interesting to isolate, e.g., because they are from the same department. The aggregation operator could be the following (with $\alpha \in[1 / \# G, 1-1 / \# G]$ ):

$$
C_{\alpha}^{G}=q_{\alpha}^{G}\left(C_{1}, \ldots, C_{K}\right)=\left\{c \in \bigcup_{k=1}^{K} C_{k}: \frac{\#\left\{k \in G: c \in C_{k} \vee\left(c^{\prime} \Rightarrow c \wedge c^{\prime} \in C_{k}\right)\right\}}{\# G} \geqslant \alpha\right\} .
$$

Similar definitions may be proposed concerning the inputs $\left(T_{\alpha}^{G}\right)$ and results $\left(R_{\alpha}^{G}\right)$.

In the identification of quasi-dominated and quasi-optimal alternatives in standalone VIP Analysis, the DMs may consider a tolerance parameter $\varepsilon$, as explained in Section 2.1. When upgrading to Group VIP Analysis, the DM's tolerance $\varepsilon$ may vary conjointly with the majority level $\alpha$. Indeed, the DMs may inquire what alternatives are quasi-dominated according to an $\alpha$-majority, noting that obtaining a quasi-dominance with a high value for $\alpha$ may require a high value for $\varepsilon$ (see an illustrative example in Section 3).

Each DM may analyze the consequences of imprecision (robust conclusions, variability of results) using his/her model and compare these consequences with $C_{\alpha}$ (or $C_{\alpha}^{G}$, for some $G$ ). This will provide each DM with an idea of where and by how much is he/she far from the group's mainstream. An important aspect here is that each group member may vary the parameters $\alpha$ (and $\varepsilon$ ) independently of the other members when performing private comparisons between his/her conclusions and $C_{\alpha}\left(\right.$ or $\left.C_{\alpha}^{G}\right)$. As an analogy, we may imagine a group of persons listening to the same music using headphones, but each one setting the volume lower or higher at his/her discretion.

\section{Illustrative example}

As an illustrative example for the methodology proposed in this paper, let us consider a classical problem analysed by Keeney and Nair in the 70s. We follow this study as reported by Roy and Bouyssou (1993), 
which consider additive instead of multiplicative aggregation. This decision situation concerned the choice of a location for a nuclear plant, faced by the Washington Public Power Supply System. Table 1 indicates the value of seven potential sites (to simplify the presentation we consider here only a subset of the alternatives) according to six value functions representing the criteria "impact on human health", "loss of salmon", "impact on other species", "impact on economy", "aesthetics" and "cost". Let us now imagine that there are four Decision Makers responsible for the decision, who agree on the single-criteria values in Table 1, but have different opinions concerning the importance of each criterion.

Using an additive value function model, criteria importance is introduced in the form of scaling constants $k_{1}, \ldots, k_{6}$, which define trade-off ratios among the value functions. For instance, if $k_{1}=3 k_{2}$, this means that one unit of value in function $v_{2}(\cdot)$ is worth three units of value in function $v_{1}(\cdot)$. Such values are often elicited by asking questions like "how much would you be willing to lose in criterion 'cost' to gain 0.1 units in criterion 'impact on human life'?". Such questions are sometimes very hard to answer in a precise way. Hence, the possibility of answering in terms of acceptable values rather than a precise value may be particularly welcome.

In our hypothetical example, let us suppose that DMs start by placing few constraints on the values they consider acceptable, although some of them are less restrictive than others:

DM1: $k_{6} \geqslant k_{5}$ (i.e., an increase of an amount $x$ in $v_{6}(\cdot)$ is more attractive than a similar increase in $v_{5}(\cdot)$ ) DM2: $k_{6} \geqslant k_{1} \geqslant k_{2} \geqslant k_{3}$

DM3: $k_{6} \geqslant k_{j}, j=1, \ldots, 5$

DM4: $k_{6} \geqslant k_{2} \geqslant k_{4} \geqslant k_{3}$.

Furthermore, without loss of generality we consider also the "normalization" constraints $k_{1}+\cdots+k_{6}=1$ and $k_{j} \geqslant 0(j=1, \ldots, 6)$. Let us define the set

$$
T=\left\{\left(k_{1}, \ldots, k_{6}\right): k_{1}+\cdots+k_{6}=1 \quad \text { and } \quad k_{j} \geqslant 0 \quad(j=1, \ldots, 6)\right\} .
$$

The combinations of values for the scaling constants each one considers acceptable (at this stage) are

$$
\begin{aligned}
& T_{1}=\left\{\left(k_{1}, \ldots, k_{6}\right) \in T: k_{6} \geqslant k_{5}\right\} \quad T_{2}=\left\{\left(k_{1}, \ldots, k_{6}\right) \in T: k_{6} \geqslant k_{1} \geqslant k_{2} \geqslant k_{3}\right\}, \\
& T_{3}=\left\{\left(k_{1}, \ldots, k_{6}\right) \in T: k_{6} \geqslant k_{j}, \quad j=1, \ldots, 5\right\} \quad T_{4}=\left\{\left(k_{1}, \ldots, k_{6}\right) \in T: k_{6} \geqslant k_{2} \geqslant k_{4} \geqslant k_{3}\right\} .
\end{aligned}
$$

Given these four sets, VIP-G can compute the minimum and maximum global value $V\left(a_{i}\right)$ attainable by each alternative, which are depicted in Table 2 (each of these values is obtained by solving a linear program). It is visible that the result concerning $a_{1}$, for instance, varies among the DMs:

DM1's range: $V\left(a_{1}\right)_{[1]} \in[0.715,0.99]$

DM2's range: $V\left(a_{1}\right)_{[2]} \in[0.732,0.98]$

Table 1

Value of seven alternatives according to each one of six criteria

\begin{tabular}{lllllll}
\hline & $v_{1}\left(a_{i}\right)$ & $v_{2}\left(a_{i}\right)$ & $v_{3}\left(a_{i}\right)$ & $v_{4}\left(a_{i}\right)$ & $v_{5}\left(a_{i}\right)$ & $v_{6}\left(a_{i}\right)$ \\
\hline$a_{1}$ & 0.715 & 0.989533 & 0.8135 & 0.7316 & 0.98 & 0.957466 \\
$a_{2}$ & 0.8 & 0.989533 & 0.8135 & 0.7145 & 0.98 & 1 \\
$a_{3}$ & 0.875 & 0.989533 & 0.802 & 0.7149 & 0.86 & 0.96799 \\
$a_{4}$ & 0.76 & 0.99782 & 0.63 & 0.5925 & 0.88 & 0.959617 \\
$a_{5}$ & 0.78 & 0.993586 & 0.469 & 0.68535 & 0.76 & 0.728686 \\
$a_{6}$ & 0.885 & 0.998013 & 0.469 & 0.56375 & 0.98 & 0.60035 \\
$a_{7}$ & 0.74 & 0.99879 & 0.7345 & 0.5725 & 1 & 0.89758 \\
\hline
\end{tabular}


Table 2

Minimum and maximum global value of the alternatives according to the different DMs

\begin{tabular}{|c|c|c|c|c|c|c|c|c|}
\hline & \multicolumn{2}{|l|}{ DM1 } & \multicolumn{2}{|l|}{ DM2 } & \multicolumn{2}{|l|}{ DM3 } & \multicolumn{2}{|l|}{ DM4 } \\
\hline & $\min V\left(a_{i}\right)$ & $\max V\left(a_{i}\right)$ & $\min V\left(a_{i}\right)$ & $\max V\left(a_{i}\right)$ & $\min V\left(a_{i}\right)$ & $\max V\left(a_{i}\right)$ & $\min V\left(a_{i}\right)$ & $\max V\left(a_{i}\right)$ \\
\hline$a_{1}$ & 0.715 & 0.99 & 0.732 & 0.98 & 0.801 & 0.976 & 0.715 & 0.98 \\
\hline$a_{2}$ & 0.714 & 1 & 0.714 & 1 & 0.832 & 1 & 0.8 & 1 \\
\hline$a_{3}$ & 0.715 & 0.99 & 0.715 & 0.968 & 0.828 & 0.979 & 0.86 & 0.979 \\
\hline$a_{4}$ & 0.593 & 0.998 & 0.593 & 0.96 & 0.727 & 0.979 & 0.76 & 0.979 \\
\hline$a_{5}$ & 0.469 & 0.994 & 0.685 & 0.834 & 0.599 & 0.861 & 0.719 & 0.861 \\
\hline$a_{6}$ & 0.469 & 0.998 & 0.564 & 0.98 & 0.535 & 0.866 & 0.6 & 0.98 \\
\hline$a_{7}$ & 0.572 & 0.999 & 0.572 & 1 & 0.735 & 0.965 & 0.74 & 1 \\
\hline
\end{tabular}

DM3's range: $V\left(a_{1}\right)_{[3]} \in[0.801,0.976]$

DM4's range: $V\left(a_{1}\right)_{[4]} \in[0.715,0.98]$

The duality between results and conclusions can be observed:

- $V\left(a_{1}\right)_{(1 / 4)} \in[0.715,0.99]$ : values in this range are supported by at least $1 / 4$ of the DMs, but $4 / 4$ of them agree that the minimum value is 0.715 ;

- $V\left(a_{1}\right)_{(2 / 4)} \in[0.715,0.98]$ : values in this range are supported by at least $2 / 4$ of the DMs, but at least $3 / 4$ of them agree that the minimum value is 0.715 ;

- $V\left(a_{1}\right)_{(3 / 4)} \in[0.732,0.98]$ : values in this range are supported by at least $3 / 4$ of the DMs, and at least $2 / 4$ of them agree that the minimum value is 0.732 ;

- $V\left(a_{1}\right)_{(4 / 4)} \in[0.801,0.976]$ : values in this range are supported by $4 / 4$ of the DMs, but only $1 / 4$ of them agree that the minimum value is 0.801 .

VIP-G also uses linear programming to compute the maximum advantage of each alternative $a_{i}$ against any other alternative $a_{j}$, i.e., $\max \left\{V\left(a_{i}, k\right)-V\left(a_{j}, k\right): k \in T_{k}\right\}(k=1, \ldots, 4)$. The result obtained by each DM is presented in Table 3(a)-(d). For instance, according to DM1, the maximum difference of value of $a_{1}$ over $a_{2}$ is 0.017 . As another example, DM2 finds that the maximum difference of value of $a_{5}$ over $a_{1}$ is -0.046 . This means that $V\left(a_{1}\right)$ is always higher than $V\left(a_{5}\right)$ given the constraints defining $T_{2}$. Alternative $a_{5}$ is hence dominated according to DM2 (and DM3 and DM4).

Some alternatives are not dominated, but quasi-dominated if the DMs allow for a small tolerance $\varepsilon$. For instance, comparing $a_{1}$ and $a_{7}$ DMs conclude:

DM1: $\max \left\{V\left(a_{7}, k\right)-V\left(a_{1}, k\right): k \in T_{1}\right\}=0.025$; hence, $a_{1} \Delta_{0.025} a_{7}$ ( $a_{1}$ quasi-dominates $a_{7}$ with a tolerance of 0.025 , according to DM1);

DM2: $\max \left\{V\left(a_{7}, k\right)-V\left(a_{1}, k\right): k \in T_{2}\right\}=0.02$; hence, $a_{1} \Delta_{0.02} a_{7}$;

DM3: $\max \left\{V\left(a_{7}, k\right)-V\left(a_{1}, k\right): k \in T_{3}\right\}<0$; hence $a_{1} \Delta a_{7}$; (a $a_{1}$ dominates $a_{7}$, according to DM3);

DM4: $\max \left\{V\left(a_{7}, k\right)-V\left(a_{1}, k\right): k \in T_{4}\right\}=0.025$; hence, $a_{1} \Delta_{0.025} a_{7}$.

This example shows the existing interplay between the tolerance $\varepsilon$ and the majority level $\alpha$. If we denote by $a_{i} \Delta_{\varepsilon(\alpha)} a_{j}$ the assertion " $a_{i}$ quasi-dominates $a_{j}$ with tolerance $\varepsilon$ for a majority of $\alpha$ ", it is obvious that one may need a higher tolerance to obtain a wider majority supporting the conclusion, as illustrated in Fig. 4: all four DMs agree that $a_{1} \Delta_{0.025(4 / 4)} a_{7}$; two out of four DMs agree that $a_{1} \Delta_{0.02(2 / 4)} a_{7}$; and one out of four DMs finds that $a_{1}$ dominates $a_{7}$ (Fig. 4).

Let us imagine that all DMs agreed that a difference of 0.025 is negligible, i.e., they all accept a tolerance $\varepsilon=0.025$. Then there would be a consensus that alternative $a_{7}$ was quasi-dominated and could be dropped 
Table 3

Pairwise comparison of maximum difference of value according to (a) DM1, (b) DM2, (c) DM3, (d) DM4

\begin{tabular}{|c|c|c|c|c|c|c|c|}
\hline & $a_{1}$ & $a_{2}$ & $a_{3}$ & $a_{4}$ & $a_{5}$ & $a_{6}$ & $a_{7}$ \\
\hline \multicolumn{8}{|l|}{ (a) } \\
\hline$a_{1}$ & & 0.017 & 0.055 & 0.184 & 0.344 & 0.357 & 0.159 \\
\hline$a_{2}$ & 0.085 & & 0.076 & 0.184 & 0.344 & 0.4 & 0.142 \\
\hline$a_{3}$ & 0.16 & 0.075 & & 0.172 & 0.333 & 0.368 & 0.142 \\
\hline$a_{4}$ & 0.045 & 0.008 & 0.008 & & 0.231 & 0.359 & 0.062 \\
\hline$a_{5}$ & 0.065 & 0.004 & 0.004 & 0.093 & & 0.128 & 0.113 \\
\hline$a_{6}$ & 0.17 & 0.085 & 0.01 & 0.125 & 0.105 & & 0.145 \\
\hline$a_{7}$ & 0.025 & 0.009 & 0.035 & 0.104 & 0.266 & 0.297 & \\
\hline Max regret & 0.17 & 0.085 & 0.76 & 0.184 & 0.344 & 0.368 & 0.145 \\
\hline \multicolumn{8}{|l|}{ (b) } \\
\hline$a_{1}$ & & 0.017 & 0.12 & 0.139 & 0.229 & 0.357 & 0.159 \\
\hline$a_{2}$ & 0.064 & & 0.12 & 0.122 & 0.271 & 0.4 & 0.142 \\
\hline$a_{3}$ & 0.085 & 0.021 & & 0.122 & 0.239 & 0.368 & 0.142 \\
\hline$a_{4}$ & 0.024 & -0.024 & 0.02 & & 0.231 & 0.359 & 0.062 \\
\hline$a_{5}$ & -0.046 & -0.029 & -0.03 & 0.093 & & 0.128 & 0.113 \\
\hline$a_{6}$ & 0 & 0 & 0.12 & 0.1 & 0.22 & & -0.009 \\
\hline$a_{7}$ & 0.02 & 0.02 & 0.14 & 0.12 & 0.24 & 0.297 & \\
\hline Max regret & 0.085 & 0.021 & 0.14 & 0.139 & 0.271 & 0.4 & 0.159 \\
\hline \multicolumn{8}{|l|}{ (c) } \\
\hline$a_{1}$ & & -0.005 & 0.055 & 0.107 & 0.287 & 0.357 & 0.109 \\
\hline$a_{2}$ & 0.064 & & 0.076 & 0.115 & 0.308 & 0.4 & 0.122 \\
\hline$a_{3}$ & 0.085 & 0.021 & & 0.104 & 0.286 & 0.368 & 0.116 \\
\hline$a_{4}$ & 0.024 & -0.016 & 0.007 & & 0.231 & 0.359 & 0.062 \\
\hline$a_{5}$ & -0.051 & -0.079 & -0.088 & -0.031 & & 0.128 & -0.005 \\
\hline$a_{6}$ & -0.045 & -0.077 & -0.057 & -0.033 & 0.066 & & -0.036 \\
\hline$a_{7}$ & -0.001 & -0.024 & 0.035 & 0.054 & 0.225 & 0.297 & \\
\hline Max regret & 0.085 & 0.021 & 0.076 & 0.115 & 0.308 & 0.4 & 0.122 \\
\hline \multicolumn{8}{|l|}{ (d) } \\
\hline$a_{1}$ & & 0 & 0.12 & 0.1 & 0.229 & 0.357 & 0.072 \\
\hline$a_{2}$ & 0.085 & & 0.12 & 0.1 & 0.271 & 0.4 & 0.102 \\
\hline$a_{3}$ & 0.16 & 0.075 & & 0.115 & 0.239 & 0.368 & 0.135 \\
\hline$a_{4}$ & 0.045 & -0.016 & 0.02 & & 0.231 & 0.359 & 0.062 \\
\hline$a_{5}$ & 0.065 & -0.02 & -0.088 & 0.02 & & 0.128 & 0.04 \\
\hline$a_{6}$ & 0.17 & 0.085 & 0.12 & 0.125 & 0.22 & & 0.145 \\
\hline$a_{7}$ & 0.025 & 0.02 & 0.14 & 0.12 & 0.24 & 0.297 & \\
\hline Max regret & 0.17 & 0.085 & 0.14 & 0.125 & 0.271 & 0.4 & 0.145 \\
\hline
\end{tabular}

from consideration for best choice. Analogously, $a_{4}$ and $a_{5}$ could also be unanimously discarded for $\varepsilon=0.025$. Only DM1 prevents $a_{6}$ from being considered quasi-dominated. This alternative could be dropped if either the group accepted a $3 / 4$ majority $(\alpha=0.75)$ or if DM1 accepted a tolerance of 0.085 . As a matter of fact, if all DMs accepted a tolerance $\varepsilon=0.085$, then $a_{2}$ would quasi-dominate all other alternatives: it is quasi-optimal for that tolerance for all the DMs.

The methodology of VIP-G can hence be used to simplify the problem, as illustrated here, by a progressive elimination of the less interesting alternatives. Despite the lack of precision and imperfect con- 


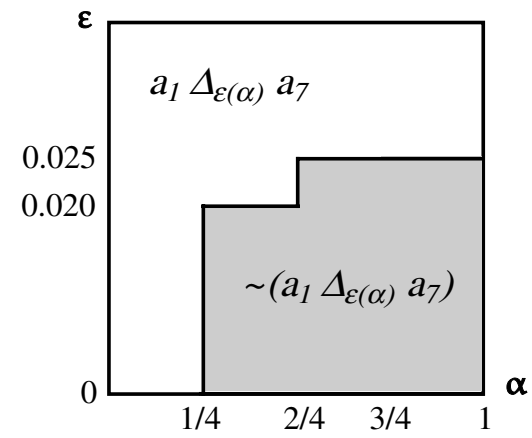

Fig. 4. Relation between tolerance and majority for the pair $(a 7, a 1)$.

sensus, that may be enough to see emerge a clear winner. Another role of this methodology should be to help each DM individually to provide more precise inputs at the same time he/she learns the results yielded by the other DMs' inputs. The group may readily appreciate where they are in agreement and where they most disagree, using this information to guide the process. However, VIP-G does not deal with the possibility of group members trying to manipulate results or sabotage consensus.

\section{A GDSS architecture for VIP-G}

Fig. 5 outlines the planned architecture for VIP-G. At its current development stage, VIP-G is able to process and aggregate the DMs' inputs, although it lacks the message passing and management capabilities that will allow its users to communicate effortlessly. It is planned to be a system based on a server to which several DMs may connect using the Internet. Indeed, this presents the advantage of allowing DMs to log in from virtually any place with access to the Internet.

Each DM uses a client application similar to the standalone VIP Analysis to perform private analyses, using the server to disclose and to gather information regarding the inputs he/she is considering and the results he/she is getting. The server's role is to manage the database of the DM's inputs, broadcasting to all the DMs (i.e. to the client applications) the inputs collected from all of them (this waiting condition may be replaced by a calendar deadline). If anonymity is required, then the server may aggregate inputs and results,

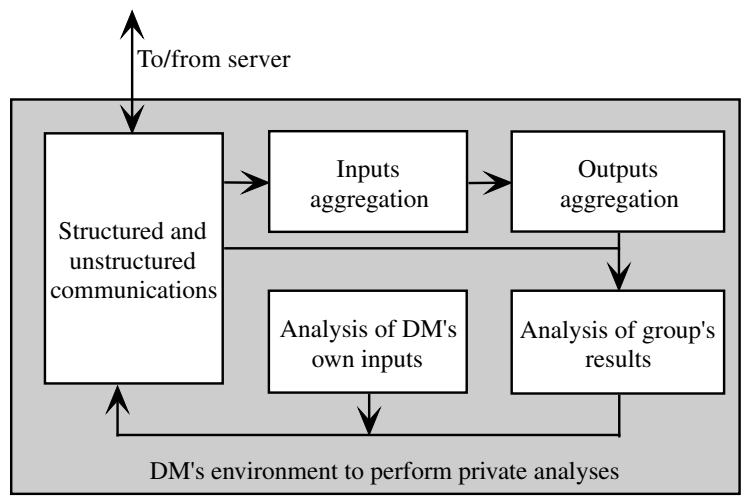

Fig. 5. VIP-G architecture: Client application. 
rather than sending the complete set of inputs or outputs to the DMs. In this case, the modules "Inputs aggregation" and "Outputs aggregation" might be located in the server, rather than the client. However, DMs would no longer be able to analyze the results of sub-groups of interest chosen by them (as proposed in Section 2.4).

The module ("Analysis of DM's own inputs" module) corresponds to the use of VIP Analysis as if its user was the sole DM. The entire interaction occurs as when using the standalone version of VIP Analysis. Individual analyses may be confronted with the DM's view of any group G's current standings ("Analysis of group's results" module), namely the results $R_{\alpha}^{G}$. This view is also personal, in that the DM may select the composition of $G$ (whole group, whole group except him/her, etc.), and may privately set the parameters for the majority level $(\alpha)$ and the tolerance $(\varepsilon)$.

The results $R_{\alpha}^{G}$ are either simply aggregated by the module "Outputs aggregation", after collecting the outputs $R_{k}(k \in G)$ currently disclosed by $G$ 's members, or computed after the module "Inputs aggregation" collects and aggregates the inputs $T_{k}(k \in G)$ currently disclosed by $G$ 's members. The conclusions $C_{\alpha}^{G}$ stem directly from the results, as explained in Section 2.4 .

Fig. 6 presents an example of the envisaged confrontation of an individual DM's perspective with the group's aggregated perspective (the data are the same used in Section 3). The chart on the left corresponds to the private analysis of the DM using the client application. The chart on the right corresponds to the aggregated outputs of a group of interest defined by that DM, consisting of him/her plus three other DMs (i.e. $K=4)$. This chart shows three ranges for each alternative: the outer one corresponds to $R_{(1 / 4)}\left(C_{(1)}\right)$; the middle one (gray) corresponds to $R_{(\alpha)}\left(C_{(1-\alpha+1 / 4)}\right)$, i.e. $R_{(3 / 4)}\left(C_{(1 / 2)}\right)$; and the inner one (black) corresponds to $R_{(1)}\left(C_{(1 / 4)}\right)$. Considering $a_{2}$, for instance, the values in the range $[0.715,0,99]$ are obtainable by at least one from the four DMs (thus, all four agree that $\left.V\left(a_{2}\right) \geqslant 0.715\right)$. The values in the range $[0.732,0.98]$ are obtained by at least three DMs (thus, $50 \%$ of the group agree that $V\left(a_{2}\right) \geqslant 0.732$ ). Finally, the values in the range $[0.801,0,976]$ are obtainable by all four DMs, but that means that only one of them would agree with the conclusion that $V\left(a_{2}\right) \geqslant 0.801$. All this information is concisely presented. If the DM changes the required majority level (using the "trackbar") then the middle ranges (gray) will also change. They will coincide with the outer ranges (white) if the majority is set at the minimum $\mathrm{R}: 25 \% / \mathrm{C}: 100 \%$; they will coincide with the inner ranges (black) if the majority is set at the maximum $\mathrm{R}: 100 \% / \mathrm{C}: 25 \%$.

Fig. 7 illustrates the presentation of the aggregation of pair-wise confrontation tables from a group of four DMs (the data are the same used in Section 3). Each cell presents three numbers: the one at the left top corresponds to corresponds to $R_{(1)} C_{(1 / 4)}$; the one at the right top corresponds to $R_{(1 / 4)}\left(C_{(1)}\right)$; the one at the middle bottom corresponds to $R_{(1 / 2)}\left(C_{(3 / 4)}\right)$. For instance, the numbers corresponding to $\left(a_{1}, a_{2}\right)$ inform that: $a_{2} \Delta_{(1 / 4)} a_{1}, a_{2} \Delta_{0,017(3 / 4)} a_{1}$, and $a_{2} \Delta_{0,017(4 / 4)} a_{1}$, i.e., if the DMs accept a tolerance of 0.017 , then it is unanimous that $a_{1}$ is dominated by $a_{2}$.

The module "Structured and unstructured communications" (Fig. 5) is responsible for all the communications between the user's working environment and the server. It receives structured messages about

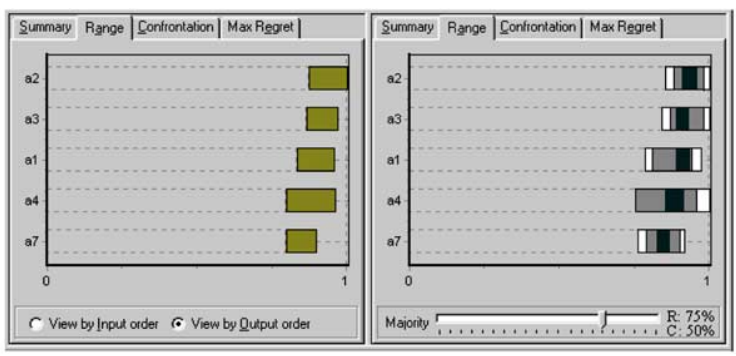

Fig. 6. Private vs. group ranges. 


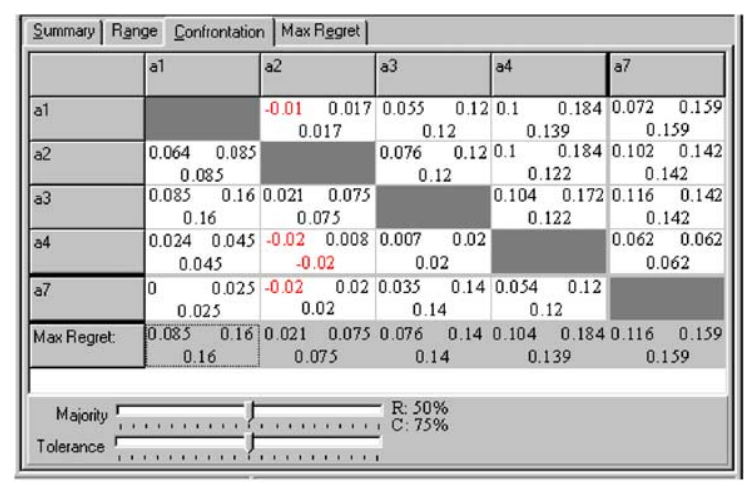

Fig. 7. Aggregation of opinions concerning dominance among alternatives.

other DMs' sets $R_{k}$ (results-passing messages) and/or $T_{k}$ (models-passing messages), which are sent to the "Outputs aggregation" and "Inputs aggregation" modules, respectively. Moreover, it may send each user's sets $R_{k}$ and/or $T_{k}$, when they wish to do so, to the other DMs. Depending on the characteristics of the group and the decision problem, a user-driven approach may suffice, or a facilitator may be needed to establish deadlines and to encourage participation. This module will also allow unstructured communications (as in e-mail) to enable informal discussions both about the decision problem (e.g., to defend an opinion, or to propose the exclusion of an alternative) and the decision-making process (e.g., to establish a deadline, or to prompt others to discuss an open issue).

\section{Summary and future research}

We started by reviewing VIP Analysis, as proposed by Dias and Clímaco. It is a methodology/software suitable to support a group of DMs meeting face-to-face around a computer, sharing an imprecisely defined model, therefore agreeing on the robust conclusions. The outputs of VIP Analysis may allow the decision process to progress, as the DMs learn about the model and the problem, postponing the elicitation questions they find difficult to answer, or prone to generate conflict. The software can be used to direct the elicitation of further information with the purpose of progressively reducing the imprecision, using these outputs to structure the dialogue among the DMs.

We have then proposed an extension that we named VIP-G, offering explicit aggregation tools, and allowing DMs to work privately and spatially distributed, while keeping the advantages mentioned in the previous paragraph. Each DM can perform private analyses, which may be confronted with his/her view of any group $G$ 's current standings, privately setting the parameters for the majority level $(\alpha)$ and the tolerance (ع). VIP-G maintains the "exploration" philosophy of VIP Analysis, not demanding precise information, and providing robust conclusions with wide support from the group (possibly with a tolerance). Not only may this prevent unnecessary conflict (there is a many-to-one correspondence between parameter values and conclusions), but it may guide the group decision-making process. Moreover, the feedback each member receives as what can accepted by the other members can facilitate issuing proposals (e.g. "can we drop $a_{7}$ from consideration?", "can we agree that $k_{1} \geqslant k_{2}$ ?") and can encourage the convergence of opinions (unfortunately, it can also facilitate manipulation, but we are assuming that all group members are genuinely trying to reach consensus).

The focus of this paper has been directed to the aggregation of results, which seemed the most promising than the aggregation of inputs (parameter values), emphasizing the duality between shared results and 
shared conclusions, and the compromise between majority $(\alpha)$ and tolerance $(\varepsilon)$. Nevertheless, we believe that the aggregation of inputs deserves further research, namely on overcoming the difficulties in using nonconvex sets resulting from the aggregation, and helping the DMs in the cases where the aggregated set of acceptable inputs is empty. Anticipating such cases, VIP-G could be complemented with tools to deal with inconsistent systems of constraints (Chinneck, 1997) to propose alternatives to restore the consistency that could be accepted by the group.

The details concerning the communications module of the proposed GDSS are still a subject for future research, both at the client level and server level. The Internet offers excellent potential to support communications, and an interesting development for the future would be to implement VIP-G clients based on a Web interface, to take advantage of the users' familiarity with browsers.

Furthermore, other decision support tools could be offered to use the knowledge about the inputs and outputs of all the DMs to compute some measures of the proximity among its members (e.g. comparing rankings, or computing distances among the centroids of $T_{1}, \ldots, T_{K}$ ) and their degree of indecisiveness (e.g. comparing the volumes of $T_{1}, \ldots, T_{K}$ ). Some of these tools might be available only to a facilitator, if there is one. Here also, there is a vast field for research.

Finally, at a more general level, there are two interesting items for future research: to study how to avoid manipulation of results (if possible), and to study whether (or how) a system like the proposed Group VIP Analysis may be used for negotiation support.

\section{Acknowledgement}

This research has been partially funded by FCT project POSI/SRI/37346/2001.

\section{References}

Aizerman, M.A., Aleskerov, F.T., 1983. Local operators in models of social choice. Systems \& Control Letters 3, 1-6.

Athanassopoulos, A.D., Podinovski, V.V., 1997. Dominance and potential optimality in MCDA with imprecise information. Journal of the Operational Research Socociety 48, 142-150.

Avis, D., Bremner, D., Seidel, R., 1997. How good are convex hull algorithms? Computational Geometry: Theory and Applications 7, 265-302.

Bana e Costa, C.A., 1986. A multicriteria decision aid methodology to deal with conflicting situations on the weights. European Journal of Operational Research 26, 22-34.

Bana e Costa, C.A., 1990. Une méthode pour l'aide à la décision en situations multicritères et multiacteurs. Sistemi Urbani 3, 301-332.

Bose, U., Paradice, D., 1999. The effects of integrating cognitive feedback and multi-attribute utility-based multicriteria decisionmaking methods in GDSS. Group Decision and Negotiation 8, 157-182.

Chinneck, J.W., 1997. Feasibility and viability. In: Gal, T., Greenberg, H.J. (Eds.), Advances in Sensitivity Analysis and Parametric Programming. Kluwer, Dordrecht, pp. 14.1-14.41.

Davey, A., Olson, D., 1998. Multiple criteria decision making models in group decision support. Group Decision and Negotiation 7, $55-77$.

Dias, L.C., Clímaco, J.N., 2000a. Additive aggregation with variable interdependent parameters: The VIP analysis software. Journal of the Operational Research Society 51, 1070-1082.

Dias, L.C., Clímaco, J.N., 2000b. ELECTRE TRI for groups with imprecise information on parameter values. Group Decision and Negotiation 9, 355-377.

Hokkanen, J., Lahdelma, R., Miettinen, K., Salminen, P., 1998. Determining the implementation order of a general plan by using a multicriteria method. Journal of Multi Criteria Decision Analysis 7, $273-284$.

Keeney, R.L., Raiffa, H., 1976. Decisions with Multiple Objectives: Preferences and Value Tradeoff. Wiley, New York.

Kersten, G., Cray, D., 1996. Perspectives on representation and analysis of negotiation: Towards cognitive support systems. Group Decision and Negotiation 5, 433-467.

Kim, S.-H., Ahn, B.S., 1997. Group decision making procedure considering preference strength under incomplete information. Computers and Operations Research 24, 1101-1112. 
Kim, S.H., Ahn, B.S., 1999. Interactive group decision making procedure under incomplete information. European Journal of Operational Research 116, 498-508.

Kim, S.H., Han, C.H., 1999. An interactive procedure for multi-attribute group decision making with incomplete information. Computers and Operations Research 26, 755-772.

Kim, S.H., Choi, S.H., Kim, J.K., 1999. An interactive procedure for multi-attribute group decision making with incomplete information: Range-based approach. European Journal of Operational Research 118, 139-152.

Lahdelma, R., Salminen, P., 2001. SMAA-2: Stochastic multicriteria acceptability analysis for group decision making. Operations Research 49, 444-454.

Lahdelma, R., Hokkanen, J., Salminen, P., 1998. SMAA-Stochastic multiobjective acceptability analysis. European Journal of Operational Research 106, 137-143.

Roy, B., Bouyssou, D., 1993. Aide Multicritère à la Décision: Methods et Cas. Economica, Paris.

Von Winterfeldt, D., Edwards, W., 1986. Decision Analysis and Behavioral Research. Cambridge University Press, New York.

Weber, M., 1987. Decision making with incomplete information. European Journal of Operational Research $28,44-57$.

Zimmermann, H.-J., 2000. An application-oriented view of modeling uncertainty. European Journal of Operational Research 122, 190-198. 